\title{
Structural-phase state of near-surface layers of VT6 titanium alloy after femtosecond laser treatment
}

\author{
M. V. Zhidkov ${ }^{\dagger, 1}$, N. A. Smirnov², J. Chen², S. I. Kudryashov², M. N. Yapryntsev ${ }^{1}$ \\ †zhidkov@bsu.edu.ru
}

${ }^{1}$ Belgorod National Research University, 85 Pobedy St., Belgorod, 308015, Russia

${ }^{2}$ P. N. Lebedev Physical Institute, RAS, 53 Leninsky Av., Moscow, 119991, Russia

\begin{abstract}
Samples from VT6 titanium alloy were surface treated with femtosecond laser pulses ( $\tau=320 \mathrm{fs}, \lambda=1030 \mathrm{~nm})$. Samples were treated both in air and in water. Surface topography, elemental composition, and structural-phase state of the surface-modified layers were studied using scanning electron microscopy and X-ray diffraction analysis. It was found that treatment with a fluence of $1.2 \mathrm{~J} / \mathrm{cm}^{2}$ leads to the formation of laser-induced periodic surface structures (LIPSS) on the surface of the VT6 titanium alloy. The period of the structures depends on the number of pulses and the processing medium: from $\sim 1010 \mathrm{~nm}$ for 1 pulse in air to $\sim 580 \mathrm{~nm}$ for 100 pulses in water. It was found that the surface structure after multi-pulse treatment exhibits superhydrophobic behavior with a contact angle of approximately 150 degrees. It was found that multi-pulse treatment both in air and in water leads to surface oxidation with the formation of $\mathrm{TiO}$ and an increase in the content of $\beta$-Ti in the near-surface layers. The magnitude of residual stresses of the first kind (macro residual stresses) in the surface layers of VT6 titanium alloy samples after femtosecond laser irradiation was estimated by the methods of X-ray diffraction analysis. The conventional $\sin ^{2} \psi$ method and the X-ray grazing incidence technique (GIXD-method) were used in this research. It was shown that, after processing with a fluence of $1.2 \mathrm{~J} / \mathrm{cm}^{2}$, tensile residual stresses with a maximum magnitude of $\sim 400 \mathrm{MPa}$ are formed in the surface layers $(\sim 3 \mu \mathrm{m})$ of the samples.
\end{abstract}

Keywords: titanium alloys, femtosecond laser treatment, phase composition, residual stresses.

УДК: $539.211,539.26$

\section{Структурно-фазовое состояние приповерхностных слоев титанового сплава ВТ6 после фемтосекундной лазерной обработки}

\author{
Жидков М.В. ${ }^{\dagger, 1}$, Смирнов Н. А. ${ }^{2}$, Чэнь Ц. ${ }^{2}$, Кудряшов С. И. ${ }^{2}$, Япрынцев М. Н. ${ }^{1}$ \\ ${ }^{1}$ Белгородский государственный национальный исследовательский университет, \\ ул. Победы, 85, Белгород, 308015, Россия \\ ${ }^{2}$ Физический институт им. П.Н. Лебедева РАН, Ленинский пр., 53, Москва, 119991, Россия
}

Проведена поверхностная обработка образцов титанового сплава ВТ6 лазерными импульсами фемтосекундной длительности ( $\tau=320$ фс, $\lambda=1030$ нм). Обработка образцов проводилась на воздухе и в воде. Методами растровой электронной микроскопии и рентгеноструктурного анализа исследовано влияние параметров обработки на топографию поверхности, элементный состав и структурно-фазовое состояние приповерхностных модифицированных слоев. Показано, что обработка с плотностью энергии одиночного импульса 1.2 Дж/см² приводит к формированию на поверхности титанового сплава ВТ6 периодических поверхностных структур. Период структур зависит от числа импульсов и среды обработки: от 1010 нм для 1 импульса на воздухе до 580 нм для 100 импульсов в воде. Показано, что многоимпульсная обработка на воздухе позволяет придавать поверхности сплава ВТ6 супергидрофобные свойства $\left(\Theta \sim 150^{\circ}\right)$. Выявлено, что многоимпульсная обработка как на воздухе, так и в воде, приводит к окислению поверхности с формированием оксида титана ТіО и увеличению доли $\beta$-Ті в приповерхностных слоях. Методами рентгеноструктурного анализа проведена количественная оценка величины остаточных напряжений 1 рода (макронапряжений) в приповерхностных слоях образцов титанового сплава ВТ6 после фемтосекундного лазерного облучения. Использовалась стандартная съемка по методу $\sin ^{2} \psi$ и анализ 
в скользящем рентгеновском пучке (grazing incidence X-ray diffraction или GIXD-метод). Показано, что после обработки с плотностью энергии 1.2 Дж/см² в приповерхностных слоях ( 3 мкм) образцов формируются растягивающие остаточные напряжения. Максимальная величина растягивающих напряжений составляет 400 МПа.

Ключевые слова: титановые сплавы, фемтосекундная лазерная обработка, фазовый состав, остаточные напряжения.

\section{1. Введение}

Одним из перспективных направлений в области создания новых технологий обработки материалов является поверхностная модификация металлов и сплавов концентрированными потоками энергии, в частности, лазерным излучением короткой и ультракороткой длительности [1], электронными $[2,3]$ или ионными пучками [4]. Развивающиеся при таком воздействии процессы, связанные с перестройкой структуры, физико-химическими превращениями и изменением топографии поверхности, зачастую позволяют придавать материалу уникальный комплекс физикохимических и механических свойств.

Известно, что использование фемтосекундных лазерных импульсов позволяет формировать на металлах и сплавах поверхностные структуры с характерными размерами элементов рельефа в очень широком диапазоне: от периодического поверхностного нанорельефа с субволновым [5] и волновым периодом (Laser Induced Periodic Surface Structure или LIPSS) [6,7] до развитого квазипериодического микрорельефа с многоуровневой шероховатостью [1]. У ряда материалов после такой обработки обнаружено проявление супергидрофобных [8] и супергидрофильных свойств [9], увеличение поглощательной способности до $100 \%$ [10], улучшение трибологических характеристик [11]. Особый интерес вызывает использование фемтосекундных лазеров для создания поверхностей с антибактериальными свойствами [12]. Антибактериальные свойства поверхностей, нанотекстурированных фемтосекундны ми лазерами, продемонстрированы для нержавеющих сталей $[13,14]$ и титановых сплавов [15-18].

Все эти факторы позволяют считать фемтосекундную лазерную обработку перспективным методом для поверхностной модификации материалов медицинского назначения, например имплантатов из титановых сплавов. Влияние фемтосекундных импульсов на топографию поверхности титановых сплавов рассмотрено в ряде работ [1,5-7], однако структурно-фазовый состав и остаточные напряжения, возникающие в приповерхностных слоях титановых сплавах при такой обработке практически не изучены (за исключением работы [19]).

В настоящей работе исследуется влияние параметров лазерной обработки на топографию поверхности, фазовый состав и остаточные напряжения приповерхностных слоев титанового сплава ВТ6.

\section{2. Материал и методика}

В качестве объекта исследований был выбран $\alpha+\beta$ титановый сплав ВТ6 (Ti-6Al-4V). Образцы подвергались термической обработке (отжигу) при $850^{\circ} \mathrm{C}$ в течение 1 часа с последующим охлаждением на воздухе. Для лазерной обработки были подготовлены образцы размером $10 \times 10$ мм. Перед лазерной обработкой проводилась механическая шлифовка и полировка образцов на установке LaboPol-5 (Struers) до «зеркального блеска» с химическим травлением в растворе ( $\left.10 \% \mathrm{HF}, 87 \% \mathrm{H}_{2} \mathrm{O}, 3 \% \mathrm{HNO}_{3}\right)$.

Образцы обрабатывали на волоконном иттербиевом лазере, генерирующим ультракороткие лазерные импульсы длительностью 320 фс на длине волны 1030 нм. Параметры обработки представлены в Табл. 1.

Топографию и элементный состав поверхности до и после обработки исследовали на растровом электронном микроскопе (РЭМ) FEI Quanta 600, оснащенном энергодисперсионным спектрометром EDAX (разрешение по Ве 0.2\%). Структуру материала в исходном состоянии исследовали методом дифракции отраженных электронов на РЭМ FEI Nova Nanosem 450.

Рентгенофазовый анализ (РФА) проводился на рентгеновском дифрактометре ARL X'TRA в фокусировке по Брегга-Брентано в диапазоне углов $2 \Theta=30-90^{\circ}$ в $\mathrm{Cu}_{\mathrm{Ka}}$-излучении. РФА в скользящем пучке проводили на рентгеновском дифрактометре Rigaku Smartlab при фиксированных углах падения рентгеновского излучения $\alpha=1,3,5,10,15^{\circ}$ в диапазоне углов $2 \Theta=30-90^{\circ}$ в $\mathrm{Cu}_{\mathrm{Ka}}$-излучении. Рассчитанные толщины исследуемых слоев $(t)$ представлены в Табл. 2.

Оценку остаточных напряжений 1 рода проводили по методу $\sin ^{2} \psi$ по смещению пика (213) при углах $\psi=0,10,20,30,40^{\circ}$ и по методу g- $\sin ^{2} \psi[20]$ в «скользящей» геометрии съемки.

Табл. 1. Режимы лазерной обработки.

Table 1. Irradiation modes used in experiment.

\begin{tabular}{|c|c|c|c|}
\hline $\begin{array}{l}\text { Режим, № } \\
\text { Mode No. }\end{array}$ & $\begin{array}{c}\text { Плотность энергии } \\
\left(F_{0}\right), \text { Дж/см² } \\
\text { Fluence }\left(F_{0}\right), \mathrm{J} / \mathrm{cm}^{2}\end{array}$ & $\begin{array}{c}\text { Число } \\
\text { импульсов }(N) \\
\text { Number of } \\
\text { pulses }(N) \\
\end{array}$ & $\begin{array}{c}\text { Среда } \\
\text { обработки/ } \\
\text { Processing } \\
\text { medium } \\
\end{array}$ \\
\hline 1 & \multirow{3}{*}{1.2} & 1 & воздух / air \\
\hline 2 & & 100 & воздух / air \\
\hline 3 & & 100 & вода / water \\
\hline
\end{tabular}

Табл. 2. Расчетная глубина проникновения рентгеновского излучения в сплав ВТ6.

Table 2. Calculated penetration depth of X-ray beam into the titanium alloy VT6.

\begin{tabular}{|c|c|c|c|c|c|c|}
\hline $\begin{array}{c}\text { Угол падения а, град. } \\
\text { Incidence angle } \alpha \text {, deg. }\end{array}$ & 1 & 3 & 5 & 10 & 15 & $\begin{array}{c}\text { Геометрия Брегга-Брентано } \\
\text { Bragg-Brentano geometry }\end{array}$ \\
\hline Глубина $t$, мкм / Depth $t, \mu \mathrm{m}$ & 0.24 & 0.72 & 1.2 & 2.3 & 3.5 & 13.7 \\
\hline
\end{tabular}




\section{3. Результаты и обсуждение}

\section{1. Структура и элементный состав поверхности}

В исходном (перед облучением) состоянии образцы титанового сплава ВТ6 имели двухфазную $(\alpha+\beta)$ структуру. Средний размер зерна $\alpha$-фазы составлял $4.6 \pm 2.6$ мкм по данным ДОЭ-анализа (Рис. 1). Объемная доля $\beta$-фазы по данным РФА составляла $~ 6 \%$.

Согласно данным РЭМ, обработка фемтосекундными лазерными импульсами приводит к изменению топографии поверхности образцов титанового сплава ВТ6. Использованная в работе плотность энергии одиночного импульса $(1.2$ Дж/см²) значительно превышает пороги откольной $(0.05$ Дж/см²) и фрагментационной (фазовый взрыв) (0.3 Дж/см²) абляции для титана [19], и уже воздействие одиночного импульса на воздухе (режим № 1) приводит к формированию на поверхности титанового сплава ВТ6 периодической поверхностной структуры с околоволновым периодом $\sim 1010 \pm 80$ нм (Рис. 2a). Воздействие 100 импульсов на воздухе с плот-

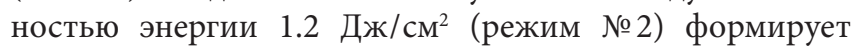
периодическую поверхностную структуру с периодом $790 \pm 100$ нм, покрытую продуктами абляции микронного и субмикронного размера (Рис. 2b). Аналогичная по параметрам обработка, но под тонким (до 2 мм) слоем дистилированной воды, позволяет получить поверхностную структуру с периодом $580 \pm 50$ нм (Рис. 2c) практически без окислившихся продуктов абляции, характерных для режима 2. Тем не менее, согласно данным ЭДА, интенсивное окисление поверхности происходит при многоимпульсной обработке как на воздухе, так

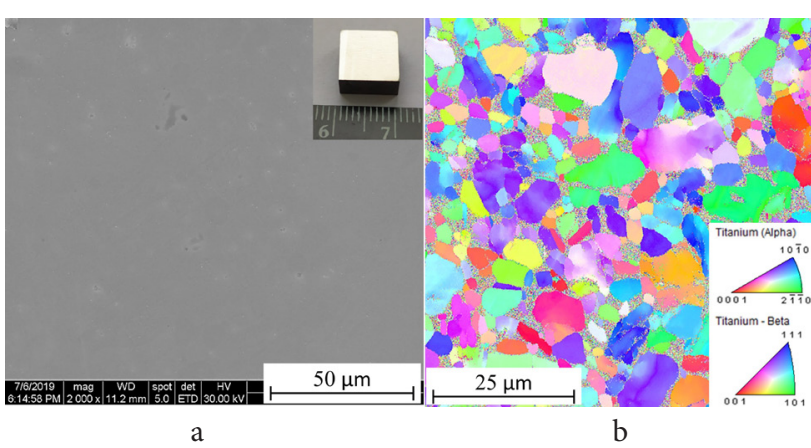

Pис. 1. (Color online) Топография (а) и структура (b) образцов титанового сплава ВТ6 в исходном состоянии.

Fig. 1. (Color online) Topography and structure of samples of VT6 titanium alloy in the initial state.

и в воде. Содержание кислорода в приповерхностном слое после обработок по режимам № 2 и № 3 составляет 23.3 и 17.3 ат.\%, соответственно.

\section{2. Свойства смачиваемости поверхности}

Формирование рельефа по режиму № 2 изменяет свойства смачиваемости водой поверхности ВТ6 с гидрофильных $\left(\Theta \sim 56^{\circ}\right)$ до сверхгидрофобных $\left(\Theta \sim 150^{\circ}\right)$. Образование периодических поверхностных структур по режимам 1 и 3 не приводит к существенному увеличению краевого угла смачивания в сравнении со свойствами исходного состояния (Рис. S1, дополнительный материал).

Проявление сверхгидрофобных свойств у титанового сплава после обработки по режиму 2, по всей вероятности может быть связано с формированием многоуровневого рельефа (периодические структуры + продукты абляции)

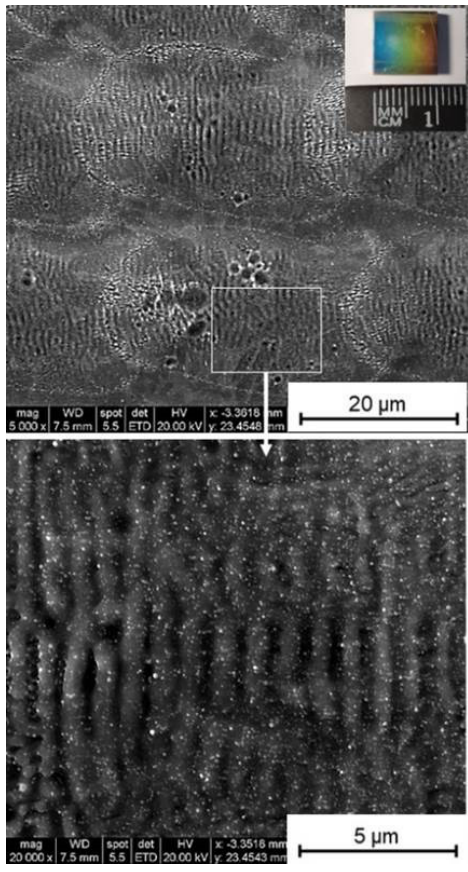

a
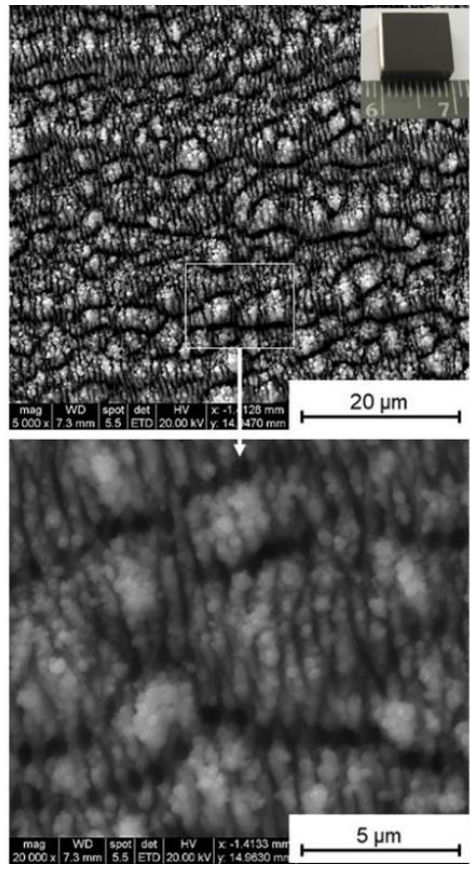

b

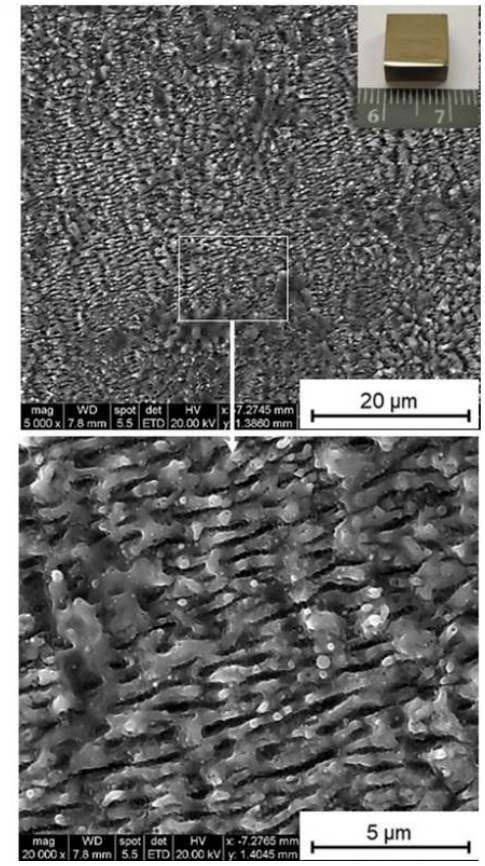

c

Puc. 2. (Color online) Топография поверхности титанового сплава ВТ6 после фемтосекундной лазерной обработки по режимам № 1 (а), № 2 (b) и № 3 (c).

Fig. 2. (Color online) Topography of surface of $6 \mathrm{Al}-4 \mathrm{~V}$ titanium alloy samples affected by fs-laser treatment in mode 1 (a), 2 (b), 3 (c). 
и реализацией гетерогенного режима смачивания [21]. Ранее сверхгидрофобные свойства титановых сплавов после фемтосекундной обработки были продемонстрированы в работах $[15,18]$.

\section{3. Фазовый состав приповерхностных слоев}

Согласно данным РФА одноимпульсная обработка по режиму 1 не приводит к изменению фазового состава приповерхностных слоев титанового сплава BT6. Фазовый состав приповерхностных слоев после многоимпульсной обработки (режим 2 и 3 ) представлен фазами: $\alpha$-Ті (ГПУ), $\beta$-Ті (ОЦК) и оксидом титана (II) $\mathrm{TiO}$ (Рис. 3 a). Объемная доля $\beta$-Ті в приповерхностных слоях после многоимпульсной лазерной обработки возрастает (Рис. 3 b). Так, согласно данным РФА, объемная доля $\beta$ - Ti в приповерхностном слое толщиной 240 нм возрастает до $25 \%$ после обработки по режиму 2 и до $15 \%$ после обработки по режиму 3. С увеличением глубины анализируемого слоя объемная доля $\beta$-фазы снижается и на глубине 1-2 мкм принимает значение, характерное для исходного состояния ( 6\%).

Увеличение объемной доли $\beta$-фазы может быть обусловлено тепловым воздействием до температур, превышающих температуру полиморфного $\alpha \rightarrow \beta$ превращения, с последующим быстрым охлаждением. Также фазовое превращение может быть вызвано изменением элементного состава приповерхностных слоев, например уменьшением содержания элементов стабилизаторов $\beta$-фазы. В частности, уменьшение содержания алюминия в приповерхностных слоях $\alpha+\beta$ титановых сплавов при фемтосекундной лазерной обработке было показано в работе [19].

\section{4. Остаточные напряжения в приповерхностных слоях}

Для анализа напряженного состояния тонких приповерхностных слоев образцов титанового сплава ВТ6

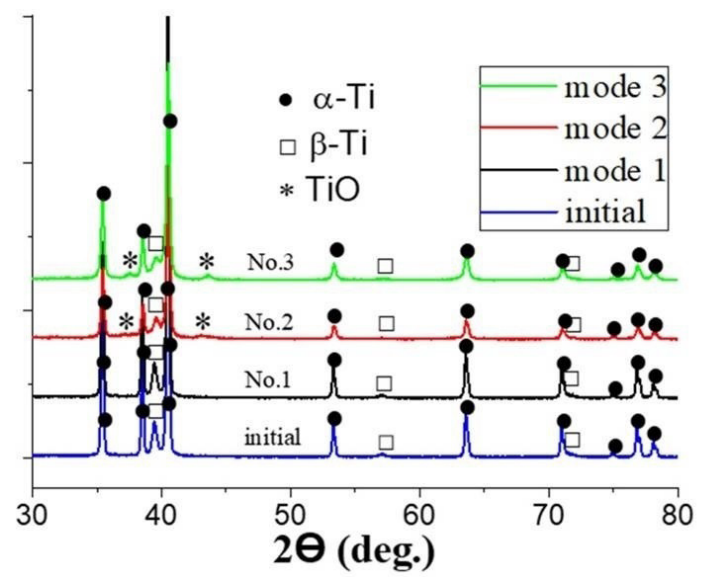

a

Pис. 3. (Color online) Рентгенограммы ( $\alpha=3^{\circ}$, толщина анализируемого слоя $t \sim 720$ нм) образцов титанового сплава ВТ6 до и после лазерной обработки (a); изменение объемной доли $\beta$-Ті в приповерхностных слоях титанового сплава ВТ6 после лазерной обработки (b).

Fig. 3. (Color online) XRD patterns $\left(\alpha=3^{\circ}\right.$, depth of the analyzed layer $t \sim 720 \mathrm{~nm}$ ) of VT6 alloy in the initial state and after fs-laser treatment (a); $\beta$-Ti content profiles in the laser-textured VT6 alloy samples (b). нами была использована рентгеновская дифракция в скользящем пучке (grazing angle incidence) и так называемый GIXD-sin ${ }^{2} \psi$ или g-sin ${ }^{2}$ метод [20,22]. В методе $\mathrm{g}-\sin ^{2} \psi$ проводилась съемка отражений с разными (hkl) для фазы $\alpha$-Ti в диапазоне углов $2 \Theta=30-90^{\circ}$ при неподвижном положении образцов и фиксированных значениях угла падения рентгеновского луча $\alpha$ (в нашем эксперименте $1,3,5,10$ и $15^{\circ}$ ). Рассчитанные глубины анализируемого слоя $t$ в зависимости от угла $\alpha$ представлены в Табл. 2 .

Связь деформации $\varepsilon_{\psi}^{h k l}$ и напряжений для двухосного напряженного состояния (метод $g(\psi, h k l))$, согласно [22] при $\varphi=0^{\circ}$ определяется выражением:

$$
\frac{\varepsilon_{0^{\circ} \psi}^{h k l}}{S_{1}^{h k l}}=g(\psi, h k l) \cdot \sigma_{1}+\left(\sigma_{1}+\sigma_{2}\right)=\left(\frac{\frac{1}{2} S_{2}^{h k l}}{S_{1}^{h k l}} \cdot \sin ^{2} \psi\right) \cdot \sigma_{1}+\left(\sigma_{1}+\sigma_{2}\right),
$$

где $S_{1}^{h k l}$ и $1 / 2 S_{2}^{h k l}$ - дифракционные упругие постоянные для плоскостей $(h k l), \psi-$ угол наклона, который для отражающей плоскости $(h k l)$ определяется как $\psi=\Theta^{h k l}-\alpha$.

Для каждой глубины анализируемого слоя $t$, представленной в Табл. 2 мы определяли $\varepsilon_{0^{\circ} \psi}^{h l}=\left(d_{\psi}^{h k l}-d_{0}^{h k l}\right) / d_{0}^{h k l}$, где $d_{0}^{h k l}$ - межплоскостные расстояния для исходного состояния (до лазерной обработки). Дифракционные упругие постоянные $\alpha$-Ti $S_{1}^{h k l}$ и $1 / 2 S_{2}^{h k l}$ рассчитывали в приложении ISODEC [23]. Из полученных данных строили графики $\varepsilon_{0^{\circ} \psi}^{h l} / S_{1}^{h k l}-g(\psi, h k l)$ (Рис. 4 a) для различных толщин исследуемого приповерхностного слоя и рассчитывали численные значения компоненты напряжений $\sigma_{1}$ и сумму напряжений $\sigma_{\varphi}=\sigma_{1}+\sigma_{2}$, лежащих в плоскости образца (Рис. 4 b).

Как видно из Рис. 4, обработка титанового сплава ВТ6 лазерными импульсами по режимам 1-3 приводит к формированию в приповерхностных слоях растягивающих напряжений. Максимальная измеренная величина напряжений $\sigma_{\varphi}$ составляет $\sim 400$ МПа. Для многоимпульсной обработки в воде (режим 3) максимальные напряжения формируются в приповерхностном слое 240 нм и непре-

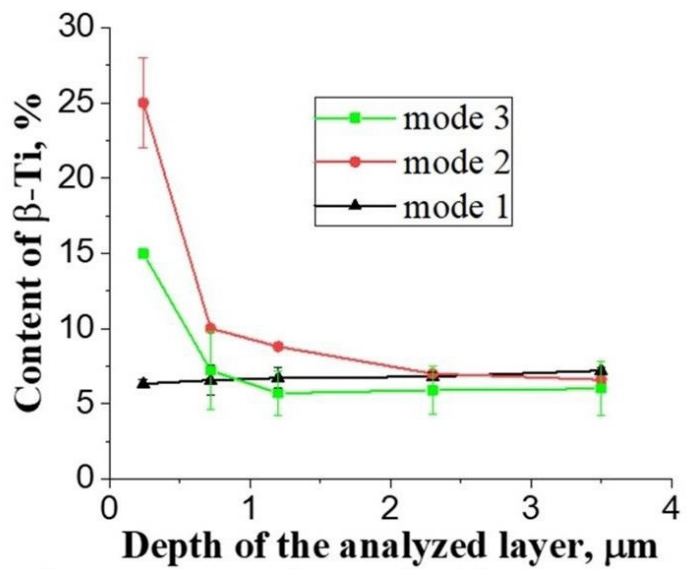

b 


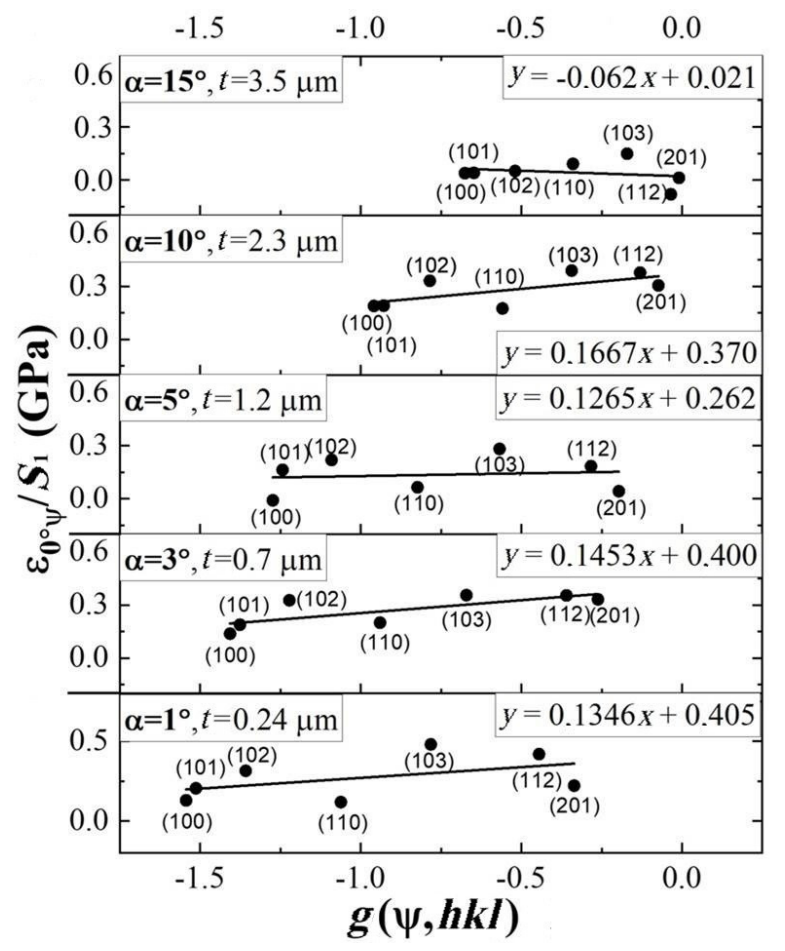

a
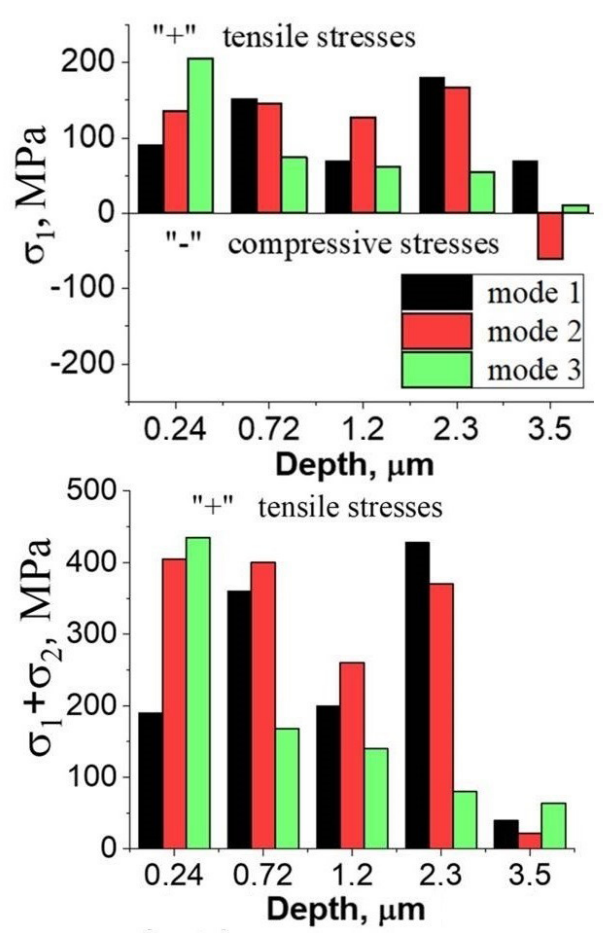

$\mathrm{b}$

Puc. 4. (Color online) Анализ макронапряжений по методу g- $\sin ^{2} \psi$ для образца титанового сплава ВТ6, обработанного по режиму № 2 (а); распределение остаточных напряжений в приповерхностных слоях образцов титанового сплава ВТ6 после обработки по режимам № 1-3 (b).

Fig. 4. (Color online) Macro stresses analysis using the g- $\sin ^{2} \psi$ method for a VT6 titanium alloy sample after femtosecond laser treatment in mode 2 (a); distribution of residual stresses in the near-surface layers of VT6 titanium alloy after femtosecond laser treatment in mode 1-3 (b).

рывно убывают с увеличением толщины анализируемого слоя. Для образцов, облученных на воздухе (режим 1 и 2) максимальные напряжения наблюдаются на больших глубинах ( 2.3 мкм) (Рис. 4 b). Согласно данным PCA, остаточные напряжения в образцах титанового сплава ВТ6 после фемтосекундной лазерной обработки по режимам 1-3 сосредоточены в тонких приповерхностных слоях толщиной 3 мкм. Анализ слоя толщиной 14 мкм с использованием стандартного метода $\sin ^{2} \psi$ фиксирует величины остаточных напряжений, близкие к нулевому значению, характерные для исходного (до лазерной обработки) состояния. Приведенные данные о глубине залегания остаточных напряжений коррелируют с результатами, полученными ранее для $\alpha+\beta$ титановых сплавов, поверхностномодифицированных одиночными фемтосекундными импульсами ( $\tau=100 \mathrm{fs}, \lambda=744 \mathrm{~nm})$ на воздухе [19].

\section{4. Выводы}

Обработка образцов титанового сплава ВТ6 фемтосекундными импульсами с плотностью энергии одиночного импульса $F_{0} \sim 1.2$ Дж/см² приводит к формированию периодических поверхностных структур. Период структур зависит от числа импульсов и среды обработки: от $\sim 1010$ нм для 1 импульса на воздухе до 580 нм для 100 импульсов в воде. Рельеф, сформированный 100 импульсами на воздухе придает поверхности титанового сплава ВТ6 сверхгидрофобные свойства $\left(\Theta \sim 150^{\circ}\right)$. Воздействие 100 импульсов как в воде, так и на воздухе приводит к окислению поверхности с формированием оксида $\mathrm{TiO}$ и увеличению доли $\beta$-Ti в приповерхностных слоях. Формирование периодических поверхностных структур при $F_{0} \sim 1.2$ Дж/см² приводит к формированию в приповерхностных слоях ( 3 мкм) растягивающих остаточных напряжений.

Дополнительный материал/Supplementary material. Электронная версия статьи содержит дополнительныи материал (Pис. S1), доступный безвозмездно на сайте журнала (lettersonmaterials.com). / The online version of this paper contains supplementary material (Fig. S1) available free of charge at the journal's Web site (lettersonmaterials.com)

Благодарности/Acknowledgements. Исследование выполнено за счет гранта Российского научного фонда (проект № 19-79-00257). / The study was carried out with a grant from the Russian Science Foundation (project No. 19-79-00257).

\section{Литература/References}

1. A. A. Ionin, S. I. Kudryashov, S. V. Makarov, A. A. Rudenko, L. V. Seleznev, D. V. Sinitsyn, E. V. Golosov, Yu. R. Kolobov, A. E. Ligachev. Appl. Surf. Sci. 284, 634 (2013). Crossref

2. Y.F. Ivanov, N. N. Koval, S. V. Gorbunov, S. V. Vorobyov, S. V. Konovalov, V. E. Gromov. Russ. Phys. J. 54, 5 (2011). Crossref

3. Yu. F. Ivanov, V.E. Gromov, S. V. Konovalov, D. V. Zagulyaev, E. A. Petrikova, A. P. Semin. Prog. Phys. Met. 19, 2 (2018). $\underline{\text { Crossref }}$ 
4. M. V. Zhidkov, A.E. Ligachev, Yu. R. Kolobov, G. V. Potemkin, G.E. Remnev. Russ. J. Non-ferrous Metals. 60, 590 (2019). Crossref

5. C.S.R. Nathala, A. Ajami, A. A. Ionin, S.I. Kudryashov, S. V. Makarov, T. Ganz, A. Assion, W. Husinsky. Opt. Express. 23, 5915 (2015). Crossref

6. J. Bonse, S. Höhm, A. Rosenfeld, J. Krüger. Appl. Phys. A. 110, 3 (2013). Crossref

7. C. Albu, A. Dinescu, M. Filipescu, M. Ulmeanu, M. Zamfirescu. Appl. Surf. Sci. 278, 347 (2013). Crossref

8. A. Y. Vorobyev, C. Guo. J. Appl. Phys. 117, 033103 (2015). Crossref

9. Yu.R. Kolobov, M.Yu. Smolyakova, A. Yu. Kolobova, A.A. Ionin, S.I. Kudryashov, S.V. Makarov, P.N. Saltuganov, D.A. Zayarny, A.E. Ligachev. Laser Phys. Lett. 11, 125602 (2014). $\underline{\text { Crossref }}$

10. A. Y. Vorobyev, V.S. Makin, C. Guo. Phys. Rev. Lett. 102, 234301 (2009). Crossref

11. J. Bonse, R. Koter, M. Hartelt, D. Spaltmann, S. Pentzien, S. Höhm, J. Krüger. Appl. Phys. A. 117, 103 (2014). $\underline{\text { Crossref }}$

12. A. Jaggessar, H. Shahali, A. Mathew, P. K. D. V. Yarlagadda. J. Nanobiotechnol. 15, 64 (2017). Crossref

13. N. Epperlein, F. Menzel, K. Schwibbert, R. Koter, J. Bonse, J. Sameith, J. Kruger, J. Toepel. Appl Surf Sci. 418, 420 (2017). $\underline{\text { Crossref }}$
14. A.A. Nastulyavichus, S.I. Kudryashov, I.N. Saraeva, N.A. Smirnov, A.A. Rudenko, E.R. Tolordava, A. A. Ionin. Laser Phys. Lett. 17, 016003 (2019). Crossref

15. E. Fadeeva, V. K. Truong, M. Stiesch, B. N. Chichkov, R. J. Crawford, J. Wang, E. P. Ivanova. Langmuir. 27, 3012 (2011). Crossref

16. V.K. Truong, H.K. Webb, E. Fadeeva, B. N. Chichkov, A.H.F. Wu, R. Lamb, J.Y. Wang, R.J. Crawford, E. P. Ivanova. Biofouling. 28, 539 (2012). $\underline{\text { Crossref }}$

17. A. Cunha, A.-M. Elie, L. Plawinski, A.P. Serro, A. M. Botelho Do Rego, A. Almeida, M.C. Urdaci, M.-C. Durrieu, R. Vilar. Appl Surf Sci. 360, 485 (2016). Crossref

18. S. Shaikh, S. Kedia, D. Singh, M. Subramanian, S. Sinha. J. Laser Appl. 31, 022011 (2019). Crossref

19. Y.R. Kolobov, E.V. Golosov, T.N. Vershinina, M. V. Zhidkov, A. A. Ionin, S. I. Kudryashov, S. V. Makarov, L. V. Seleznev, D. V. Sinitsyn, A. E. Ligachev. Appl. Phys. A. 119, 241 (2015). Crossref

20. S. J. Skrzypek, A. Baczmanski, W. Ratuszek, E. Kusoir. J. App. Cryst. 34, 427 (2001). Crossref

21. L. B. Boinovich, A. M. Emelyanenko. Russ. Chem. Rev. 77, 7 (2008). $\underline{\text { Crossref }}$

22. U. Welzel, J. Ligot, P. Lamparter, A.C. Vermeulen, E. J. Mittemeijer. J. Appl. Cryst. 38, 1 (2005). Crossref

23. T. Gnäupel-Herold. J. Appl. Cryst. 45, 573 (2012). ㄷossref 